\title{
ADAPTACIÓN Y ESTABILIDAD DEL RENDIMIENTO DE GRANO DE GENOTIPOS DE MIJO PERLA (Pennisetum americanum L. Leeke) EN SAN LUIS POTOSÍ, MÉXICO
}

\section{ADAPTATION AND GRAIN YIELD STABILITY OF PEARL MILLET (Pennisetum americanum L. Leeke) GENOTYPES IN SAN LUIS POTOSÍ, MÉXICO}

\author{
José A. Hernández Alatorre ${ }^{1^{*}}$ y Francisco Zavala García ${ }^{2}$
}

\begin{abstract}
${ }^{1}$ Campo Experimental San Luis, Instituto Nacional de Investigaciones Forestales, Agrícolas y Pecuarias. Carr. San Luis Potosí-Matehuala km. 14.5, Soledad de Graciano Sanchez, San Luís Potosí, S.L.P. Teléfono: 01 (444) 85243 16. Fax 01 (444) 81391 51. Facultad de Agronomía de la UANL, Carretera Zuazua-Marín, Km 17, Marín, N.L. México.

*Autor para correspondencia (hernandez.jose@inifap.gob.mx.)
\end{abstract}

\section{RESUMEN}

En el Estado de San Luis Potosí el principal problema para la producción de granos es la poca disponibilidad de agua de riego y las lluvias escasas e irregulares, por lo que se requieren cultivos alternativos que se ajusten a la escaza humedad disponible. El objetivo de esta investigación fue determinar la adaptación y estabilidad del rendimiento de tres híbridos enanos y cuatro variedades de porte normal de mijo perla (Pennisetum americanum $\mathbf{L}$. Leeke). La evaluación se desarrolló en seis ambientes de riego y cinco de temporal o secano en el Altiplano, la Zona Media y la Planicie Huasteca del Estado de San Luis Potosí, ubicados a 1880, 1000 y 40 msnm, respectivamente, durante 2006 y 2007. Los genotipos se establecieron en un diseño de bloques completos al azar con tres repeticiones en cada ambiente. Se registró el rendimiento de grano, días a madurez, altura de planta, excersión, índice de cosecha, número de semillas por panícula y peso de semilla. Mediante el método de Eberhart y Russell se estimaron los parámetros de estabilidad $b_{i}$ y $S_{d i}^{2}$. En condiciones de riego, la Zona Media fue el mejor ambiente de producción, seguida de la Planicie Huasteca y el Altiplano; mientras que en temporal el mejor ambiente fue la Planicie Huasteca, seguida de la Zona Media y Altiplano. Los híbridos mostraron mayor estabilidad de rendimiento que las variedades en ambientes de riego; en contraste en temporal las variedades fueron más estables que los híbridos. La variedad 'ICMV-93191' y el híbrido precoz '68AI x 086R' mostraron buena estabilidad de rendimiento en ambientes desfavorables, por lo que representan alternativas viables para reducir las pérdidas de cosechas en las regiones semiáridas del estado.

Palabras clave: Pennisetum americanum, cultivos alternativos, ambientes contrastantes, rendimiento de grano, estabilidad.

\section{SUMMARY}

In the state of San Luis Potosí, México, the main limitation for grain crops production is the scarce water availability in the irrigated areas, and the poor and erratic rainfall distribution in the rainfed agriculture. For this reason, alternative crops that could fit limited water resources are required. The objective of this study was to determine the adaptation and grain yield stability of three dwarf hybrids and four normal plant height pearl millet (Pennisetum americanum L. Leeke) open pollinated varieties. Research was carried out at six irrigation and five rainfed environments in the highlands, midlands and lowlands of the state of San Luis Potosí, located at 1880, 1000 and $40 \mathrm{~m}$ of altitude, respectively in 2006 and 2007. The genotypes were planted in a randomized complete block design with three replications in each environment. Grain yield, days to maturity, plant height, exertion, harvest index, seed number and seed weight were registered. Estimates of stability parameters for $b_{i}$ and $S_{d i}^{2}$ grain yield were estimated with the Eberhart and Russell methodology. Under irrigated conditions, midlands were the best environments for pearl millet production, followed by lowlands and highlands; while for rainfed environments lowlands were better than midlands and highlands. At the irrigated environments the pearl millet hybrids had higher grain yield stability than open pollinated varieties; in contrast, at the rainfed environments the open pollinated varieties were more stable than hybrids. The variety 'ICMV-93191' and the early hybrid ' $68 \mathrm{AI} \times$ 086R', which were yield stable throughout the unfavorable environments, could be good alternatives to reduce risks of crop losses in rainfed highlands of the state.

Index words: Pennisetum americanum, alternative crops, contrasting environments, grain yield, stability.

\section{INTRODUCCIÓN}

El mijo perla (Pennisetum americanum L. Leeke) es originario de los trópicos semiáridos de África y se encuentra en India, Pakistán, Bangladesh, Burma y Sri Lanka donde es alimento básico de los pobladores de áreas semiáridas y áridas (Maiti y López, 1995). En Argentina y Estados Unidos se cultiva principalmente para forraje (Pale et al., 2003). Este cultivo prospera en temporadas de lluvia errática y en áreas con disponibilidad limitada de agua de riego, en donde otros cultivos como maíz (Zea mays L.) y sorgo (Sorghum bicolor L. Moench) no prosperan o producen rendimientos bajos (Muchow, 1989). Según Maiti y López (1995), en la zona de Sahel de África Occidental los genotipos precoces de mijo pueden 
cultivarse para grano con una precipitación media anual de 250 a $300 \mathrm{~mm}$, mientras que en la misma área el sorgo requiere al menos de $350 \mathrm{~mm}$ de lluvia.

En México casi no se conoce el cultivo de mijo perla, ni su tecnología de producción y potencial productivo. Los estudios experimentales de adaptación son esporádicos. Maiti y Gómez (1990) evaluaron variedades de mijo perla en varias fechas de siembra en el Estado de Nuevo León y observaron que la siembra de julio fue mejor que la de agosto o septiembre, y que los rendimientos de grano fueron mayores que los que se obtienen en la India con las mismas variedades. Torres et al. (1994) evaluaron 10 variedades de mijo perla en la región norte de Tamaulipas y aplicaron las labores de preparación del suelo y durante el desarrollo del cultivo similares a las del cultivo del sorgo en siembras de temporal o secano. Los autores encontraron que el rendimiento de grano promedio fue de $2385 \mathrm{~kg} \mathrm{ha}^{-1}$, en un intervalo de 1991 a $2919 \mathrm{~kg} \mathrm{ha}^{-1}$; la variedad 'EX-Born' fue la de mayor rendimiento, seguida por la variedad 'WC-C75' con $2647 \mathrm{~kg} \mathrm{ha}^{-1}$.

El Estado de San Luis Potosí cuenta con 6.3 millones de hectáreas, $63 \%$ de las cuales tienen problemas de sequía (lluvia escasa y de distribución irregular) y se ubican en su mayoría en las regiones conocidas como Altiplano y Zona Media del Estado (Hernández et al., 2007). En esas condiciones, los cultivos tradicionales (maíz y sorgo) que sustentan gran parte de la actividad agropecuaria, sólo cubren $43 \%$ y $67 \%$ de sus usos consuntivos estimados entre los 450 y $550 \mathrm{~mm}$ para maíz, y 457 y $510 \mathrm{~mm}$ para sorgo (Villanueva et al., 2001). Lo anterior ocasiona un alto índice de siniestralidad y rendimientos más bajos que la media nacional.

En la búsqueda de cultivos alternativos de mayor precocidad que se ajusten a la limitada disponibilidad de agua, se ha evaluado la producción de forraje del mijo perla con expectativas favorables por su buen rendimiento y calidad (Hernández et al., 2007); sin embargo, se desconoce su potencial como cultivo para grano. El objetivo del presente trabajo fue determinar la adaptación y estabilidad del rendimiento de grano de híbridos y variedades de mijo perla en condiciones de riego y temporal, en las principales regiones agroecológicas del Estado de San Luís Potosí. Esta información permitiría evaluar la factibilidad de promover el establecimiento del mijo perla como un cultivo de alternativa exitoso en el estado.

\section{MATERIALES Y MÉTODOS}

El estudio se llevó a cabo en seis ambientes en riego y cinco en temporal en el estado. En riego, los ambientes (localidad $\mathrm{x}$ año) fueron los siguientes: Ébano (Planicie Huasteca) ciclos otoño-invierno (O-I) del 20052006 y 2006-2007; Río Verde (Zona Media) y Soledad de Graciano Sánchez (Altiplano) ciclos primavera-verano (PV) del 2006 y 2007. En temporal los ambientes fueron: Ébano, P-V 2007 y Cerritos (Zona Media) y Villa Hidalgo (Altiplano) P-V 2006 y 2007. Las características geográficas, climáticas y edáficas de los ambientes de prueba se presentan en el Cuadro 1.

Se evaluaron las variedades de polinización libre de porte normal 'ICMV-88903', 'ICTP- 8203', 'ICMV-221' e 'ICMV-93191' introducidas del ICRISAT (International Crops Research Institute for the Semi-arid Tropics) y los híbridos enanos '68AI x 086R', '59052M x 9Rm x 4Rm' y ' $8401 \mathrm{M}$ x 68A4Rw' introducidos de la Universidad de Nebraska-Lincoln, EE. UU.

Las variedades 'ICMV-88903' e 'ICTP-8203' son de generación antigua liberadas en 1988 en la India (Rai et al., 1990); la variedad 'ICMV-221' fue liberada también en la India en 1993 (Witcombe et al., 1997), como una alternativa de alto rendimiento para sustituir a la variedad 'ICTP-8203' en todas las regiones productoras de la India, excepto en las de precipitación menor a $400 \mathrm{~mm}$ anuales; 'ICMV-93191' es una variedad experimental que se caracteriza por tener grano de color crema, más apropiada que las otras variedades para elaborar rotis (tortilla hindú) o tortillas mexicanas. Los híbridos que se evaluaron fueron producidos mediante el sistema $A_{1}$ de esterilidad nuclearcitoplásmica masculina en la Universidad de Nebraska, como alternativas para regiones secas de ese estado en donde la producción de sorgo es inestable por problemas de sequía.

Se utilizó un diseño experimental de bloques completos al azar con tres repeticiones en cada ambiente. La parcela experimental fue de dos surcos de $0.6 \mathrm{~m}$ de ancho y $5.0 \mathrm{~m}$ de longitud, y la parcela útil los dos surcos centrales de $4.0 \mathrm{~m}$ de longitud. En el Cuadro 2 se describe el manejo agronómico en cada ambiente de prueba. El agua de riego se cuantificó con un medidor volumétrico Mc Propeller® en Soledad, y con vertedores en Ébano y Río Verde. En los ambientes de temporal la precipitación fue registrada en mililitros en un "bote rustico o chilero" y multiplicada por el factor 0.0527 para obtener la lluvia en mm de cada evento pluvial (Jasso, 2005; Com. pers.) ${ }^{1}$.

\footnotetext{
${ }^{1}$ Cesario Jasso Chaverría. Investigador del Programa 'Productividad de Agrosistemas', Campo Experimental San Luis-Centro de Investigación Regional del Noreste-INIFAP-SAGARPA.
} 
Cuadro 1. Características geográficas, climáticas y edáficas de los ambientes de riego y de temporal o secano en los que se evaluaron genotipos de mijo perla. San Luis Potosí. 2006-2007.

\begin{tabular}{|c|c|c|c|c|c|c|c|}
\hline Ambiente & Localidad & Año & $\begin{array}{l}\text { Ubicación } \\
\text { geográfica }\end{array}$ & $\begin{array}{c}\text { Altitud } \\
\text { (m) }\end{array}$ & $\begin{array}{c}\text { Temperatura } \\
\text { mínima-maxima } \\
\text { promedio } \neq \\
\left({ }^{\circ} \mathrm{C}\right) \\
\end{array}$ & $\begin{array}{c}\text { Precipitación } \\
\text { media } \neq \\
\text { anual } \\
(\mathrm{mm})\end{array}$ & Características de suelo \\
\hline \multirow[t]{2}{*}{ Riego } & $\begin{array}{l}\text { Soledad de } \\
\text { G. S. } \\
\text { (Altiplano). }\end{array}$ & 2006 & $22^{\circ} 11^{\prime} 00^{\prime \prime} \mathrm{N}$ & 1882 & $\dagger 13.0-24.7$ & 332 & Migajón arenoso, $\mathrm{pH}=8.3$ \\
\hline & & 2007 & $100^{\circ} 56^{\prime} 00^{\prime \prime} \mathrm{O}$ & & & & \\
\hline \multirow[t]{2}{*}{ Riego } & $\begin{array}{l}\text { Río Verde } \\
\text { (Zona } \\
\text { Media). }\end{array}$ & 2006 & $21^{\circ} 56^{\prime} 00^{\prime \prime} \mathrm{N}$ & 987 & $17.1-30.0$ & 488 & Migajón arcilloso arenoso, $\mathrm{pH}=8.4$ \\
\hline & & 2007 & $99^{\circ} 59^{\prime} 00^{\prime \prime} \mathrm{O}$ & & & & \\
\hline Riego & $\begin{array}{l}\text { Ébano } \\
\text { (Planicie } \\
\text { Huasteca. } \\
\text { Otoño- } \\
\text { Invierno). }\end{array}$ & $\begin{array}{c}2005-2006 \text { y } \\
2006-2007\end{array}$ & $22^{\circ} 12^{\prime} 46^{\prime \prime} \mathrm{N}$ & 40 & $16.4-27.7$ & 863 & Arcillo limoso, $\mathrm{pH}=8.2$ \\
\hline Temporal & $\begin{array}{l}\text { Ébano } \\
\text { (Primavera- } \\
\text { Verano). }\end{array}$ & 2007 & $98^{\circ} 21^{\prime} 41^{\prime \prime} \mathrm{O}$ & 40 & $22.8-32.7$ & 863 & Arcillo limoso, $\mathrm{pH}=8.2$ \\
\hline \multirow[t]{2}{*}{ Temporal } & $\begin{array}{l}\text { Villa } \\
\text { Hidalgo } \\
\text { (Altiplano). }\end{array}$ & 2006 & $22^{\circ} 51^{\prime} 00^{\prime \prime} \mathrm{N}$ & 1600 & $11.3-28.1$ & 352 & Arcilloso, $\mathrm{pH}=8.0$ \\
\hline & & 2007 & $100^{\circ} 41^{\prime} 00^{\prime \prime} \mathrm{O}$ & & & & \\
\hline \multirow[t]{2}{*}{ Temporal } & $\begin{array}{l}\text { Cerritos } \\
\text { (Zona } \\
\text { Media). }\end{array}$ & 2006 & $22^{\circ} 25^{\prime} 47^{\prime}, \mathrm{N}$ & 1178 & $15.2-29.2$ & 656 & Migajón arcilloso, $\mathrm{pH}=8.2$ \\
\hline & & 2007 & $100^{\circ} 17^{\prime} 13^{\prime \prime} \mathrm{O}$ & & & & \\
\hline
\end{tabular}

Fuente: Medina et al. (2005). † Durante el periodo de cultivo. $\ddagger$ Promedios del periodo 1961-2001.

Cuadro 2. Manejo agronómico aplicado en los ambientes de riego y de temporal. San Luis Potosí. 2006-2007.

\begin{tabular}{|c|c|c|}
\hline Actividades & Ambientes de riego & Ambientes de temporal \\
\hline Preparación del terreno & Maquinaria & Maquinaria \\
\hline $\begin{array}{l}\text { Periodo de siembra (día-mes- } \\
\text { año) }\end{array}$ & $\begin{array}{l}\text { Ébano (27-01-06 y 23-01-07), Río Verde ( } 21-04-06 \text { y } 31- \\
\text { 05-07) y Soledad de G. S. (31-05-06 y 14-06-07). }\end{array}$ & $\begin{array}{l}\text { Cerritos (07-06-06 y } 27-06-07) \text {, Villa Hidalgo (02-08-06 } \\
\text { y 11-07-07) y Ébano (15-09-07). }\end{array}$ \\
\hline Fertilización (N-P-K) & $120-60-00$ & $40-20-00$ \\
\hline Densidad (plantas/ha) & 550000 & 250000 \\
\hline Control de plagas & $\begin{array}{l}\text { Una aplicación de Lorsban } 480 \mathrm{E} \circledast 1.0 \mathrm{~L} \mathrm{ha}^{-1} \text {. Control de } \\
\text { gusano cogollero. Cipermetrina } \circledast 0.25 \mathrm{~L} \mathrm{ha}^{-1} \text {. Control de } \\
\text { chinche verde (Nezara viridula). }\end{array}$ & $\begin{array}{l}\text { Una aplicación de Cipermetrina }{ }^{\circledR} 0.25 \mathrm{~L} \mathrm{ha}^{-1} \text {. Control de } \\
\text { chinche verde (Nezara viridula). }\end{array}$ \\
\hline Escardas & Dos escardas a los 20 y $40 \mathrm{~d}$ después de la siembra. & Una escarda $30 \mathrm{~d}$ después \\
\hline Cosecha & $\begin{array}{l}\text { A los } 108,87 \text { y } 130 \text { d después de la siembra en promedio } \\
\text { en Ébano, Río Verde y Soledad. }\end{array}$ & $\begin{array}{l}\text { A los } 93,130 \text { y } 125 \text { d después de la siembra en promedio } \\
\text { en Ébano, Cerritos, y Villa Hidalgo. }\end{array}$ \\
\hline
\end{tabular}

El rendimiento de grano se obtuvo a la madurez; todas las panículas de la parcela útil se cosecharon a mano, y se les registró su peso en campo; en una submuestra de 10 panículas se determinó el porcentaje de grano, y la humedad del grano se determinó con un aparato Steinlite Electronic Tester ${ }^{\circledR}$, Model G; el rendimiento se ajustó a $12 \%$ de humedad. Los días a madurez fisiológica se contaron desde la siembra al día en que $50 \%$ de las plantas presentaron la capa negra en la base del grano. La altura de planta se midió del suelo a la punta de la panícula, y la longitud de excersión como lo distancia de la base de la panícula a la inserción de la hoja bandera del tallo principal; el índice de cosecha se calculó como el cociente del peso seco del grano entre el peso seco total de la planta. Cinco panículas al azar se desgranaron y sus semillas se contaron con una máquina Count-A-Pak® seed totalizer mod. 77-10/B, y posteriormente se pesaron. Se obtuvo el promedio del número de semillas por panícula y al dividir el peso de semilla entre el número de semillas se obtuvo el peso de semilla individual, en miligramos.

Se aplicó un análisis de varianza conjunto convencional para cada variable de los regímenes ambientales de riego y de temporal, por separado. Para la separación de medias se usó la prueba de Tukey $(\mathrm{P} \leq 0.05)$. A los datos de rendimiento se les aplicó el modelo de parámetros de estabilidad propuesto por Eberhart y Russell (1966) con el paquete estadístico elaborado por Olivares (1994). El modelo estadístico fue:

$$
y_{i j}=\mu_{i}+b_{i} I_{j}+S_{i j}
$$


Donde: $y_{i j}=$ Media del rendimiento de la variedad $i$ en el ambiente $j ; \mu_{i}=$ Media del rendimiento de la variedad $i$ sobre todos los ambientes; $b_{i}=$ Coeficiente de regresión que mide la respuesta de la variedad $i$ en varios ambientes; $I_{j}=$ Índice ambiental obtenido como la media del rendimiento de todas las variedades en el ambiente $j$, menos la media general; $S_{i j}=$ Desviación de la regresión de la variedad $i$ en el ambiente $j$.

El parámetro $b_{i}$ es el coeficiente de regresión que mide la respuesta de un genotipo al cambiar las condiciones ambientales. El parámetro $S_{d i}^{2}$ ofrece una medida de variabilidad interpretada como falta de estabilidad y mide la consistencia o inconsistencia de esa respuesta. Un genotipo es considerado estable si su coeficiente de regresión es igual a 1.0 y las desviaciones de regresión $\left(S_{d i}^{2}\right)$ son iguales a cero. El análisis proporciona pruebas de hipótesis para determinar las dos condiciones de estabilidad, así como la presencia de diferencias de medias entre los genotipos (Rodríguez et al., 2002). La clasificación de los genotipos se hizo de acuerdo con la descripción utilizada por Carballo y Márquez (1970) en función de los valores obtenidos de los parámetros $\left(b_{i} \mathrm{y}\right.$ $S_{d i)}^{2}$.

\section{RESULTADOS Y DISCUSIÓN}

Medias ambientales. Hubo diferencias ( $\mathrm{P} \leq 0.01)$ entre ambientes y genotipos para todas las variables en estudio (Cuadro 3). La interacción genotipo ambiente no fue significativa para rendimiento, pero sí para días a madurez e índice de cosecha. La heterogeneidad entre ambientes estuvo relacionada principalmente con diferencias en temperatura, tipo de suelo y régimen de humedad durante el ciclo del cultivo. En los ambientes de riego, el agua aplicada más la precipitación durante el ciclo del cultivo, varió de $300 \mathrm{~mm}$ en Ébano (2006-2007) a $581 \mathrm{~mm}$ en Soledad (2007) (Cuadro 4). Río Verde fue el mejor ambiente con un rendimiento medio de los dos años de prueba, superior en 65 y $38 \%$ al rendimiento medio de Soledad y Ébano, respectivamente. Se observó que a mayor temperatura del ambiente correspondió una más rápida madurez de los genotipos; en Río Verde ésta se presentó en promedio 35 d más temprano que en Soledad.

En temporal, el rango de precipitación fue de $150 \mathrm{~mm}$ en Cerritos (2006) hasta $382 \mathrm{~mm}$ en la misma localidad (2007), lo que evidencia gran variación de precipitación entre años para un mismo sitio (Cuadro 4). En Ébano, con una mejor distribución de la precipitación (datos no presentados), se obtuvo un rendimiento de grano superior en dos y cuatro veces al obtenido en Cerritos y Villa Hidalgo, respectivamente, lo que indica la buena adaptación del cultivo a este ambiente. Cerritos fue excepcionalmente seco en 2006 con un periodo sin lluvia de $40 \mathrm{~d}$ después de la emergencia de las plantas, por lo que la panícula del tallo principal sufrió daños severos. La cosecha no se perdió totalmente, ya que al reanudarse las lluvias los hijuelos o tallos secundarios lograron producir panícula y rendimientos aceptables, lo cual representó una característica favorable del cultivo en respuesta al periodo de sequía. Las altas temperaturas aceleraron el desarrollo del cultivo; en cambio, la sequía lo retrasó; particularmente en Cerritos 2006.

Cuadro 3. Medias para las variables sometidas al análisis combinado de siete genotipos de mijo perla en seis ambientes de riego y cinco de temporal o secano, y significancias de las fuentes de variación. San Luis Potosí. 2006-2007.

\begin{tabular}{|c|c|c|c|c|c|c|c|c|c|c|}
\hline \multirow[t]{2}{*}{ Genotipo } & \multicolumn{2}{|c|}{ Rendimiento $\left(\mathrm{kg} \mathrm{ha}^{-1}\right)$} & \multicolumn{2}{|c|}{ Días a madurez } & \multicolumn{2}{|c|}{ Altura de planta $(\mathrm{cm})$} & \multicolumn{2}{|c|}{ Excersión $(\mathrm{cm})$} & \multicolumn{2}{|c|}{ Índice de cosecha } \\
\hline & Riego & Temporal & Riego & Temporal & Riego & Temporal & Riego & Temporal & Riego & Temporal \\
\hline ‘59052M x 9R x 4Rm’ & $3278 \mathrm{~d}$ & $1788 \mathrm{~b}$ & $93 \mathrm{~d}$ & $101 \mathrm{~b}$ & $106 \mathrm{c}$ & $105 \mathrm{~b}$ & $19.7 \mathrm{a}$ & $15.7 \mathrm{a}$ & $0.34 \mathrm{bc}$ & $0.34 \mathrm{bc}$ \\
\hline ‘68AI x 086R' & $3999 \mathrm{~cd}$ & $1995 \mathrm{ab}$ & $91 \mathrm{~d}$ & $100 \mathrm{~b}$ & $106 \mathrm{c}$ & $102 \mathrm{~b}$ & $17.3 \mathrm{a}$ & $15.7 \mathrm{a}$ & $0.38 \mathrm{ab}$ & $0.38 \mathrm{ab}$ \\
\hline '8401M x 68A4R4w' & $5099 \mathrm{ab}$ & $2525 a b$ & $104 \mathrm{a}$ & $107 \mathrm{a}$ & $106 \mathrm{c}$ & $99 \mathrm{~b}$ & $13.7 \mathrm{ab}$ & $12.7 \mathrm{~b}$ & $0.41 \mathrm{a}$ & $0.38 \mathrm{a}$ \\
\hline 'ICMV-221' & 6089 a & $2120 a b$ & $102 a b c$ & $108 \mathrm{a}$ & $180 \mathrm{a}$ & $153 \mathrm{a}$ & $7.9 \mathrm{bc}$ & $6.2 \mathrm{c}$ & 0.31 cde & $0.27 \mathrm{~d}$ \\
\hline 'ICMV-88903' & $6074 \mathrm{a}$ & $2437 a b$ & $101 \mathrm{bc}$ & $108 \mathrm{a}$ & $186 \mathrm{a}$ & $155 \mathrm{a}$ & $7.4 \mathrm{c}$ & $6.7 \mathrm{c}$ & $0.30 \mathrm{de}$ & $0.30 \mathrm{dc}$ \\
\hline 'ICMV-93191' & $5358 \mathrm{ab}$ & 2682 a & $103 \mathrm{ab}$ & 107 a & $184 \mathrm{a}$ & $157 \mathrm{a}$ & $8.1 \mathrm{bc}$ & $6.9 \mathrm{c}$ & $0.27 \mathrm{e}$ & $0.28 \mathrm{~d}$ \\
\hline 'ICTP-8203' & $4518 \mathrm{bc}$ & $2133 a b$ & $100 \mathrm{c}$ & $106 \mathrm{a}$ & $163 \mathrm{~b}$ & $149 \mathrm{a}$ & $8.3 \mathrm{bc}$ & $6.7 \mathrm{c}$ & $0.34 \mathrm{bcd}$ & $0.31 \mathrm{dc}$ \\
\hline Medias & 4936 & 2240 & 99 & 105 & 147 & 132 & 11.8 & 10.1 & 0.33 & 0.32 \\
\hline Ambiente & $<0.01$ & $<0.01$ & $<0.01$ & $<0.01$ & $<0.01$ & $<0.01$ & $<0.01$ & $<0.01$ & $<0.01$ & $<0.01$ \\
\hline Genotipo & $<0.01$ & $<0.01$ & $<0.01$ & $<0.01$ & $<0.01$ & $<0.01$ & $<0.01$ & $<0.01$ & $<0.01$ & $<0.01$ \\
\hline Genotipo x Ambiente & 0.07 & 0.08 & $<0.01$ & $<0.01$ & $<0.01$ & 0.06 & 0.24 & $<0.01$ & $<0.01$ & $<0.01$ \\
\hline
\end{tabular}

Promedios con la misma letra en una columna son estadísticamente iguales (Tukey, 0.05). 
Cuadro 4. Lámina de riego y precipitación recibidas durante el ciclo del cultivo por ambiente de prueba, y medias para rendimiento, días a madurez, altura de planta, longitud de exersión e índice de cosecha, del análisis conjunto de siete genotipos de mijo perla. San Luis Potosí. 20062007.

\begin{tabular}{|c|c|c|c|c|c|c|}
\hline Ambiente & $\begin{array}{c}\text { Riegos }+\underset{(\mathrm{mm})}{\text { precipitación }} \\
\end{array}$ & $\begin{array}{c}\text { Rendimiento } \\
\text { grano } \\
\left(\mathrm{kg} \mathrm{ha}^{-1}\right) \\
\end{array}$ & $\begin{array}{c}\text { Días a } \\
\text { madurez }\end{array}$ & $\begin{array}{l}\text { Altura de planta } \\
(\mathrm{cm})\end{array}$ & $\begin{array}{l}\text { Excersión } \\
(\mathrm{cm})\end{array}$ & $\begin{array}{l}\text { Índice de } \\
\text { cosecha }\end{array}$ \\
\hline \multicolumn{7}{|l|}{ Riego } \\
\hline $\begin{array}{l}\text { Ébano 2005-2006 } \\
\text { (Planicie Huasteca) }\end{array}$ & 450 & 4706 & 84 & 120 & $\mathrm{nr} *$ & $\mathrm{nr}$ \\
\hline $\begin{array}{l}\text { Ébano 2006-2007 } \\
\text { (Planicie Huasteca) }\end{array}$ & 300 & 4508 & 94 & 144 & 13.7 & 0.29 \\
\hline $\begin{array}{l}\text { Río Verde } 2006 \\
\text { (Zona Media) }\end{array}$ & 450 & 5712 & 86 & 166 & $\mathrm{nr}$ & $\mathrm{nr}$ \\
\hline $\begin{array}{l}\text { Río Verde } 2007 \\
\text { (Zona Media) }\end{array}$ & 550 & 7045 & 83 & 184 & 8.7 & 0.34 \\
\hline $\begin{array}{l}\text { Soledad de G. S. } 2006 \\
\text { (Altiplano) }\end{array}$ & 530 & 4288 & 116 & 124 & $\mathrm{nr}$ & $\mathrm{nr}$ \\
\hline $\begin{array}{l}\text { Soledad de G. S. } 2007 \\
\text { (Altiplano) } \\
\text { Temporal }\end{array}$ & 581 & 3437 & 132 & 146 & 12.9 & 0.38 \\
\hline $\begin{array}{c}\text { Ébano } 2007 \\
\text { (Planicie Huasteca) }\end{array}$ & 304 & 4426 & 82 & 144 & 12.7 & 0.32 \\
\hline $\begin{array}{l}\text { Cerritos } 2006 \\
\text { (Zona Media) }\end{array}$ & 150 & 2326 & 133 & 115 & 9.3 & 0.41 \\
\hline $\begin{array}{l}\text { Cerritos } 2007 \\
\text { (Zona Media) }\end{array}$ & 382 & 2230 & 83 & 144 & 7.8 & 0.33 \\
\hline $\begin{array}{l}\text { Villa Hidalgo } 2006 \\
\text { (Altiplano) }\end{array}$ & 188 & 1167 & 109 & 129 & 10.4 & 0.35 \\
\hline $\begin{array}{l}\text { Villa Hidalgo } 2007 \\
\text { (Altiplano) }\end{array}$ & 163 & 1052 & 120 & 126 & 10.1 & 0.21 \\
\hline
\end{tabular}

nr* Dato no registrado.

Las medias de todas las características agronómicas fueron mayores en los ambientes favorables que en los desfavorables, pero las diferencias ambientales fueron menos evidentes que en el rendimiento de grano. En Ébano, las medias de altura de planta, excersión e índice de cosecha fueron $18 \mathrm{~cm}, 2.6 \mathrm{~cm}$ y 0.11 superiores a las medias en Villa Hidalgo, en donde la sequía ocurrió en la etapa de floración del cultivo en los dos años de evaluación.

Estabilidad del rendimiento. En el análisis de parámetros de estabilidad se encontraron diferencias entre genotipos ( $\mathrm{P} \leq 0.01)$, lo que indica amplia variabilidad genética (Cuadro 5). No hubo diferencias significativas en la interacción genotipo $\mathrm{x}$ ambiente para los datos de riego y de temporal por separado, como ya había sido observado en el análisis de varianza convencional (Cuadro 3). Esta tendencia general representa la ventaja de poder seleccionar genotipos de buena estabilidad para ambientes específicos (riego y temporal), como lo sugieren los datos de De León et al. (2005). En contraste, en el análisis conjunto de riego más temporal se observó que tanto la interacción genotipo x ambiente como la diferencia de los genotipos por su coeficiente de regresión con los índices ambientales, fueron significativos $(\mathrm{P} \leq 0.05)$ (Cuadro 5).

La estabilidad de los genotipos dependió de los regímenes ambientales involucrados. Con base en la interpretación de los estimadores del coeficiente de regresión $\left(b_{i}\right)$ y desviaciones de regresión $\left(S_{d i}^{2}\right)$ y sus combinaciones para los ambientes en riego, se identificaron como genotipos estables $\left(b_{i}=1.0 ; S_{\mathrm{di}}^{2}=0\right)$ a los híbridos '59052M x 9R x 4Rm', '68AI x 086R' y '8401M x 68A4R4w' y a la variedad 'ICTP-8203' (Cuadro 6). Las variedades 'ICMV-221' e 'ICMV-88903' fueron superiores $(\mathrm{P} \leq 0.05)$ con rendimientos medios de poco más de $6000 \mathrm{~kg} \mathrm{ha}^{-1}$, pero debido a sus altas y significativas desviaciones de regresión fueron clasificadas como variedades inconsistentes $\left(b_{i}=1.0 ; S_{\text {di }}^{2}>0\right)$. La variedad 'ICMV-93191' sobresaliente también en rendimiento, presentó un mejor comportamiento en ambientes desfavorables y consistente $\left(b_{i}<1.0 ; S_{\mathrm{di}}^{2}=0\right)$.

Los rendimientos obtenidos en este trabajo son superiores a los reportados por Mahalakshmi et al. (1987) quienes en condiciones de riego obtuvieron rendimientos de 2500 a $2900 \mathrm{~kg} \mathrm{ha}^{-1}$ en los trópicos semiáridos de la India; y a los consignados por Maiti y Gómez (1990) de $3015 \mathrm{~kg} \mathrm{ha}^{-1}$ con su mejor genotipo ('ICMS-7707') en la mejor fecha de siembra (29 de julio) en Marín, Nuevo León. Los altos rendimientos comparativos obtenidos en este trabajo sugieren que el potencial productivo de los genotipos de reciente formación se ha mejorado sustancialmente y que su adaptación en la región de estudio es aceptable. 
Cuadro 5. Cuadrados medios y significancia de las fuentes de variación del análisis combinado de estabilidad de siete genotipos de mijo perla en seis ambientes de riego y cinco de temporal San Luis Potosí. 2006-2007.

\begin{tabular}{|c|c|c|c|c|c|c|}
\hline \multirow[t]{2}{*}{ Fuente de variación } & \multicolumn{2}{|c|}{ Riego } & \multicolumn{2}{|c|}{ Temporal } & \multicolumn{2}{|c|}{ Riego + Temporal } \\
\hline & GL & $\mathrm{CM}$ & GL & $\mathrm{CM}$ & GL & $\mathrm{CM}$ \\
\hline Genotipos & 6 & $6405675^{* *}$ & 6 & $507688^{* *}$ & 6 & $4913579 * *$ \\
\hline Genotipos x Ambiente (lineal) & 6 & 565091 & 6 & 212135 & 6 & $1434936^{*}$ \\
\hline Desviación conjunta & 28 & $654024 *$ & 21 & 220727 & 63 & $63491976 * *$ \\
\hline Error conjunto & 81 & 342909 & 70 & 158668 & 151 & 151257499 \\
\hline
\end{tabular}

$\overline{\mathrm{GL}}=$ Grados de libertad; $\mathrm{CM}=$ Cuadrados medios. ${ }^{* *}$ Significancia estadística a 0.05 y 0.01 de probabilidad de error, respectivamente.

Cuadro 6. Rendimiento medio $\left(\mathrm{kg} \mathrm{ha}^{-1}\right)$, coeficientes de regresión $\left(b_{i}\right)$ y desviaciones de regresión $\left(S^{2}{ }_{d i}\right)$ de siete genotipos de mijo perla en seis ambientes de riego y cinco de temporal. San Luis Potosí. 2006-2007.

\begin{tabular}{lccccccccc}
\hline Genotipo & \multicolumn{3}{c}{ Riego } & \multicolumn{3}{c}{ Temporal } & \multicolumn{3}{c}{ Riego-Temporal } \\
\cline { 2 - 9 } & $\mathrm{kg} \mathrm{ha}^{-1}$ & $b_{i}$ & $S_{d i}^{2}$ & $\mathrm{~kg} \mathrm{ha}^{-1}$ & $b_{i}$ & $S_{d i}^{2}$ & $\mathrm{~kg} \mathrm{ha}^{-1}$ & $b_{i}$ & $S_{d i}^{2}$ \\
\hline '59052Mx 9R x 4Rm' & $3321 \mathrm{~d}$ & 0.824 & 75675 & $1788 \mathrm{~b}$ & 0.982 & 129656 & $2579 \mathrm{~d}$ & $0.713^{*}$ & 141601 \\
'68AI x 086R' & $3999 \mathrm{~cd}$ & 0.831 & 523333 & $1995 \mathrm{ab}$ & 0.794 & $336455^{*}$ & $3088 \mathrm{~cd}$ & 0.774 & $298078^{*}$ \\
'8401M x 68A4R4w' & $5100 \mathrm{ab}$ & 1.216 & 297713 & $2525 \mathrm{ab}$ & $1.281^{*}$ & -124591 & $3929 \mathrm{ab}$ & 1.082 & 124216 \\
'ICMV-221' & $6089 \mathrm{a}$ & 0.824 & $661998^{*}$ & $2119 \mathrm{ab}$ & 0.847 & -23530 & $4285 \mathrm{a}$ & 1.193 & $624018^{* *}$ \\
'ICMV-88903' & $6074 \mathrm{a}$ & 1.312 & $731395^{*}$ & $2443 \mathrm{ab}$ & 0.935 & -52694 & $4421 \mathrm{a}$ & 1.252 & $365881^{*}$ \\
'ICMV-93191' & $5358 \mathrm{ab}$ & $0.694 *$ & -250219 & $2682 \mathrm{a}$ & 1.151 & 262341 & $4142 \mathrm{a}$ & 0.957 & 20545 \\
'ICTP-8203' & $4636 \mathrm{bc}$ & 1.300 & 137916 & $2134 \mathrm{ab}$ & 1.011 & -93223 & $3364 \mathrm{bc}$ & 1.029 & 67001 \\
Media & 4939 & 1.000 & & 2241 & 1.000 & & 3695 & 1.000
\end{tabular}

Promedios con la misma letra en una columna son estadísticamente iguales (Tukey, 0.05$) .{ }^{*},{ }^{* *}$ Significancia estadística a 0.05 y 0.01 de probabilidad de error, respectivamente; $b_{i} *, * *$ Diferentes a $1.0 ; S^{2} d i * * *$ Mayores de cero.

La diferencia en rendimiento medio de grano entre híbridos y variedades en los ambientes de riego fue significativa $(\mathrm{P} \leq 0.01)$. Contrario a lo esperado, el rendimiento medio de las variedades de polinización libre fue superior al rendimiento medio de los híbridos en 48, 41 y 39 \% en Ébano, Río Verde y Soledad, respectivamente. Estas diferencias en rendimiento se debieron a que el número de semillas por panícula de las variedades fue superior $(\mathrm{P} \leq 0.05)$ al de los híbridos en 57, 47 y $64 \%$, en Ébano, Río Verde y Soledad, respectivamente. En el peso de semilla no se observaron diferencias significativas entre híbridos y variedades.

La diferencia entre localidades para el número de semillas por panícula fue significativa $(\mathrm{P} \leq 0.05)$; en variedades, la media entre localidades fue de 1924, 3452 y 2330 semillas por panícula en Ébano, Río Verde y Soledad, respectivamente. Estas diferencias en el número de semillas por panícula explican los rendimientos de grano superiores obtenidos en Río Verde, comparados con Ébano y Soledad (Cuadro 4). Los resultados sugieren que Río Verde posee el clima y suelo adecuados para un buen desarrollo del mijo perla en riego, mientras las altas temperaturas de Ébano durante el desarrollo del cultivo parecen acortar el periodo de llenado de grano aunque se reduce el número de semillas por panícula; en Soledad (ubicada a $1882 \mathrm{msnm}$, Cuadro 1), las temperaturas bajas al final del ciclo reducen la magnitud de los componentes de rendimiento. Estos datos coinciden con lo señalado por Maiti y López (1995), en el sentido que los limites de adaptación del cultivo están entre los 0 a los 1600 msnm y que altitudes superiores pudieran restringir la productividad del cultivo debido a temperaturas bajas.

Los híbridos presentaron en promedio $65 \mathrm{~cm}$ menos altura de planta, $9 \mathrm{~cm}$ más excersión y 0.07 más índice de cosecha que las variedades de polinización libre (Cuadro 3). Las características de altura de planta baja y uniforme combinada con una amplia excersión y un bajo porcentaje de acame, han sido introducidas en los híbridos modernos para facilitar la cosecha mecánica del grano (Pale et al., 2003). La disponibilidad de estos nuevos materiales que facilitan su cosecha mecánica, puede generar una mayor aceptación del mijo perla como cultivo comercial alternativo (Presterl y Weltzien, 2003).

Con los rendimientos medios por genotipo y los estimadores del coeficiente de regresión e índices ambientales se estimaron las líneas de regresión de dos híbridos y dos variedades (Figura 1). Tales líneas muestran 
que el potencial de rendimiento de los híbridos ' $68 \mathrm{AI} \times$ 086R' y ' $8401 \mathrm{M}$ x 68A4R4w', clasificados como estables, es de casi 5000 y $7000 \mathrm{~kg} \mathrm{ha}^{-1}$ en ambientes favorables. En dos localidades de Nebraska, Estados Unidos, en condiciones de riego el híbrido '68AI x 086R' produjo 4310 y $5240 \mathrm{~kg} \mathrm{ha}^{-1}$ (Maman et al., 2004).

La respuesta de los genotipos en condiciones de temporal fue diferente a la de riego. Los materiales estables resultaron ser en su mayoría variedades de polinización libre, mientras que en riego fueron los híbridos. Las variedades 'ICMV-221', 'ICMV-88903', 'ICMV-93191' e 'ICTP-8203', además del híbrido '59052M x 9R x 4Rm', fueron clasificados como estables, mientras que '68AI $x$ 086R' mostró un buen comportamiento en todos los ambientes pero fue inconsistente, y ' $8401 \mathrm{M} \times 68 \mathrm{~A} 4 \mathrm{R} 4 \mathrm{w}$ ' tuvo la mejor respuesta en ambientes favorables y consistente $\left(b_{i}>1.0\right.$; $\left.S_{\text {di }}^{2}=0\right)$ (Cuadro 6). La variedad 'ICMV-93191', además de mostrar estabilidad a través de los ambientes de temporal, tuvo un rendimiento medio sobresaliente.

La diferencia en el rendimiento medio de grano entre híbridos y variedades en los ambientes de temporal fue significativa ( $\mathrm{P} \leq 0.01)$ (Cuadro 3). El rendimiento medio de las variedades fue superior al de los híbridos en 11 y 40 $\%$ en Ébano y Cerritos, respectivamente; en Villa Hidalgo la diferencia no fue significativa. Al igual que en riego, estas diferencias de comportamiento de los genotipos se debieron a que el número de semillas por panícula de las variedades de polinización libre fue superior $(\mathrm{P} \leq 0.05)$ al de los híbridos en 51 y $37 \%$, en Ébano y Cerritos, respectivamente. En peso de semilla no se observaron diferencias significativas entre híbridos y variedades para estas localidades. En Villa Hidalgo, el ambiente más desfavorable, el número de semilla por panícula de las variedades superó al de los híbridos en $59 \%$; en cambio, el peso de semilla de los híbridos superó a las variedades en $15 \%$, lo que sugiere un efecto fisiológico compensatorio ya que a menor número de semillas hay mayor peso de las mismas (como en lo híbridos), y a mayor número de semillas hay menor peso de semilla (como en las variedades de polinización libre).

La diferencia entre localidades en el número de semillas fue significativa para las variedades; la media entre localidades fue de 2310, 2245 y 1363 semillas por panícula en Ébano, Cerritos y Villa Hidalgo, respectivamente. Estas diferencias en semillas por panícula explican los mayores rendimientos de grano en Ébano, comparados con Cerritos y Villa Hidalgo (Cuadro 4). Los resultados sugieren que en temporal, la localidad de Ébano posee el clima (precipitación) y suelo adecuados para un buen desarrollo del mijo perla, mientras que en Cerritos y Villa Hidalgo las escasas e irregulares precipitaciones afectan significativamente el rendimiento de grano cuyo principal componente, número de semillas, es limitado por la sequía predominante.

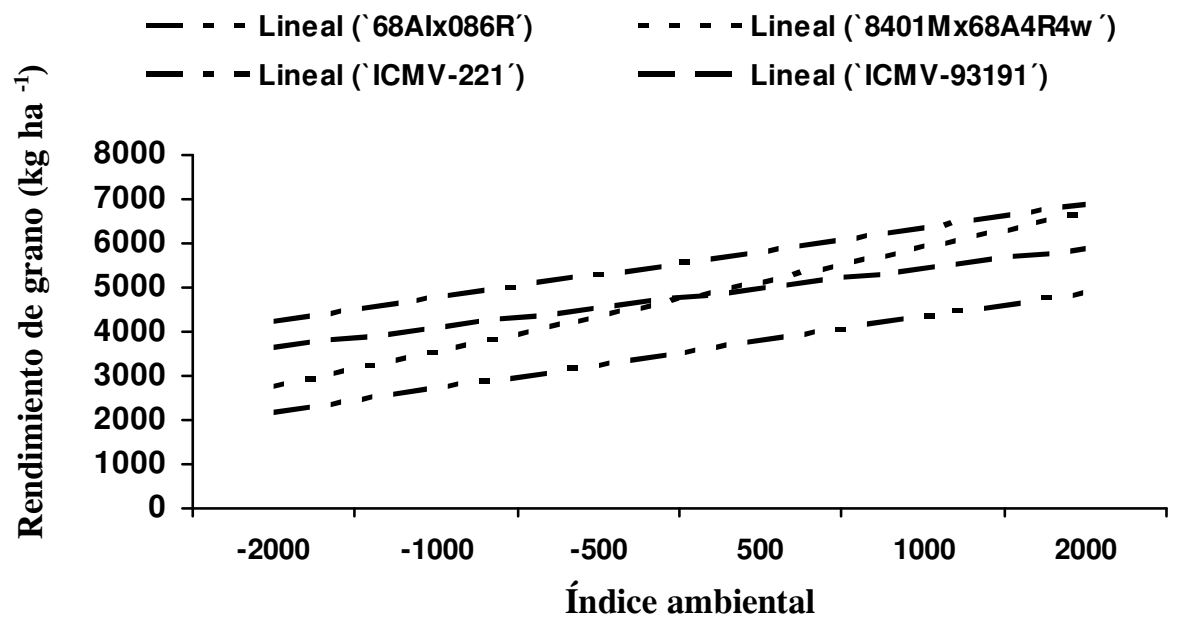

Figura 1. Rendimiento medio esperado para dos híbridos ('68AI x 086R' y '8401M x 68A4R4w') y dos variedades ('ICMV-221' e 'ICMV-93191') de mijo perla en ambientes de riego. San Luis Potosí. 2006 y 2007. 
En la Figura 2 se muestra el rendimiento esperado de dos variedades estables en temporal ('ICMV-93191' e 'ICMV-221') comparadas con dos híbridos. Se aprecia que estas variedades tienen un rendimiento potencial de 1000 $\mathrm{kg} \mathrm{ha}^{-1}$ en ambientes desfavorables y que responden positivamente en los ambientes favorables donde producen hasta 4500 y $3500 \mathrm{~kg} \mathrm{ha}{ }^{-1}$, respectivamente. Los rendimientos obtenidos en los ambientes favorables en este trabajo son similares a los reportados por Pale et al. (2003) quienes en condiciones de buen temporal reportaron rendimientos de 3100 a $5320 \mathrm{~kg} \mathrm{ha}^{-1}$ con el híbrido '68AI x 086R', en fechas de siembra sub-óptimas y óptimas, respectivamente, en el Estado de Nebraska.

La clasificación de genotipos por su estabilidad, con los datos conjuntos de riego más temporal, fue más semejante a la condición de riego que a temporal (Cuadro 6). Los genotipos fueron clasificados en dos categorías principales: genotipos estables ('8401M x 68A4R4w', 'ICMV-93191' e 'ICTP-8203'), y genotipos de buena respuesta en todos los ambientes pero inconsistentes ('68AI x 086R', 'ICMV221' e 'ICMV-88903'). El híbrido '59052M x 9R x 4Rm' resultó de mejor respuesta en ambientes desfavorables y fue consistente; sin embargo, sus bajos rendimientos lo ubican como uno de los genotipos menos deseables del grupo. Por el contrario, la variedad 'ICTP-8203', una de las variedades más antiguas del grupo y que fue liberada en 1988 en la India (Rai et al., 1990), fue el único material que mostró estabilidad a través de los ambientes de riego, temporal y conjunto (Cuadro 6), con un comportamiento estable en ambientes desfavorables y buena sensibilidad al mejoramiento ambiental. Esta variedad se caracteriza por ser de semilla grande (datos no presentados) y podría ser germoplasma valioso para seleccionar individuos segregantes, si se supone que a través de selección es posible mejorar el rendimiento y mantener estabilidad, como sugieren Mejía y Molina (2003).
Entre los genotipos precoces sobresalieron el híbrido '68AI x 086R' y la variedad 'ICMV-93191', que presentaron la madurez a los 100 y 107 d en promedio (Cuadro 3), y de buen comportamiento en fechas tardías en los ambientes desfavorables (Villa Hidalgo 2006 y 2007, Altiplano), ya que con sólo $180 \mathrm{~mm}$ de lluvia registraron un rendimiento medio de 1460 y $1220 \quad \mathrm{~kg} \mathrm{ha}^{-1}$, respectivamente (datos no presentados). El comportamiento de los genotipos destacados ('68 AI x 086R' e 'ICMV-93191') en condiciones de menor precipitación, fue debido a su mayor peso de semilla (12.20 y $9.81 \mathrm{mg} / \mathrm{semilla}$, respectivamente) y a un valor de mediano a alto del número de semillas por panícula (963 y 1186 semillas/panícula, respectivamente), comparados con el testigo ('ICMV-221') que presentó 7.74 $\mathrm{mg} /$ semilla y 1268 semillas/panícula, respectivamente.

Varios investigadores han destacado la importancia del componente peso de semilla en el rendimiento de los cultivos en condiciones de temporal (Fisher y Wilson, 1975; Fisher y Palmer, 1980). Eastin y Zavala (2002), en pruebas de campo con sorgo, indican que no sólo el número de semillas es importante, sino que el peso de semilla determina la estabilidad de genotipos al pasar de un ambiente rico a uno pobre. Estos resultados confirman además lo reportado por Maiti y López (1995), en el sentido que genotipos precoces de mijo perla pueden producir grano con sólo $250 \mathrm{~mm}$ de lluvia y con los de Pale et al. (2003) en cuanto a que por su buena tolerancia al calor y a la sequía, este cultivo podría ser una buena alternativa para segundas siembras o siembras tardías. Vittar et al. (1990) reportaron que el mijo perla superó al sorgo en estaciones de crecimiento de poca lluvia en Hyderabad, India, y que el mijo perla fue de rendimiento más estable en suelos con problemas de erosión y precipitación errática.

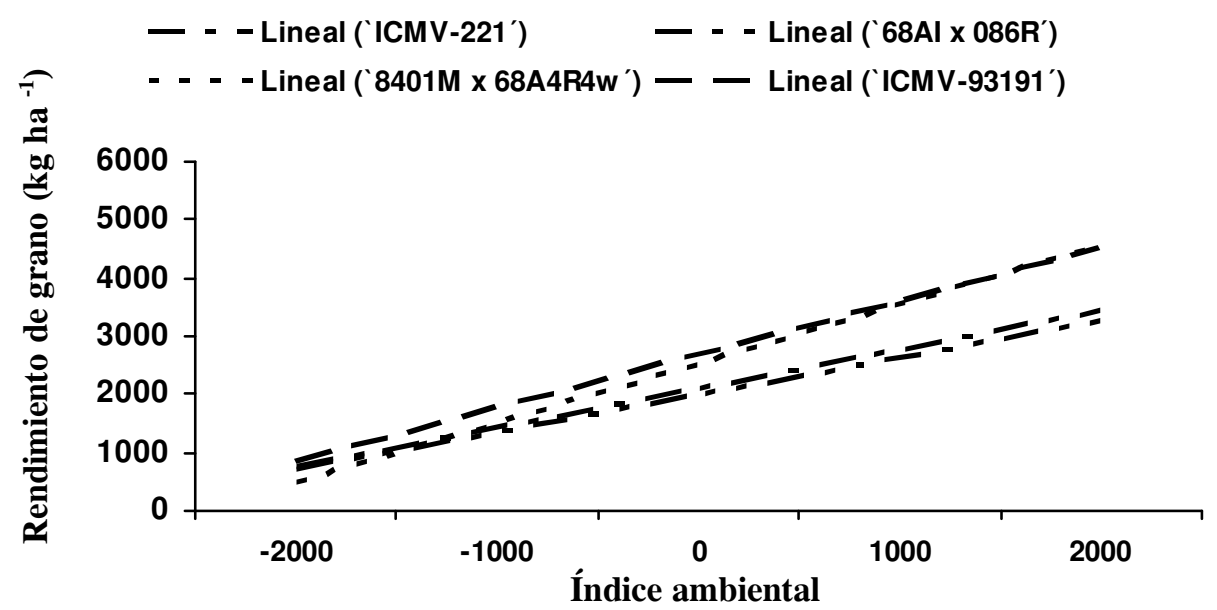

Figura 2. Rendimiento medio esperado para dos híbridos ('68AI x 086R' y '8401M x 68A4R4w') y dos variedades ('ICMV-221' e 'ICMV-93191') de mijo perla en ambientes de temporal. San Luis Potosí. 2006 y 2007. 
En el presente trabajo, aún en los ambientes desfavorables se cosechó grano. La siembra de otros cultivos de mayores requerimientos hídricos, usualmente conduce a pérdidas en las condiciones de manejo y escasa precipitación de los ambientes desfavorables en las cuales se llevó a cabo el presente estudio. Por lo anterior, se estima que el mijo perla pudiera producir cosecha en una superficie de impacto cercana a 100 mil hectáreas de bajo a mediano potencial productivo para los cultivos de maíz y sorgo en el estado de San Luis Potosí.

\section{CONCLUSIONES}

En condiciones de riego el potencial de rendimiento del mijo perla fue mayor en ambientes de la Zona Media, seguido de la Planicie Huasteca y el Altiplano, en San Luis Potosí. En temporal o secano, en respuesta a una mejor distribución de la precipitación y temperaturas calidas, el cultivo se adaptó mejor en la Planicie Huasteca, seguido de la Zona Media y del Altiplano al final.

En ambientes de riego los híbridos mostraron mayor estabilidad de rendimiento que las variedades, mientras que en temporal las variedades fueron más estables que los híbridos. Debido a su mayor adaptación general y su capacidad para producir más semillas por panícula en ambientes contrastantes, las variedades de polinización libre presentaron un rendimiento de grano superior a los híbridos.

En los ambientes desfavorables del Altiplano y Zona Media, la variedad 'ICMV-93191' y el híbrido '68AI x 086R', representan alternativas viables para reducir el riesgo de siniestralidad en una superficie estimada de 100 mil hectáreas de esta región semiárida del Estado de San Luis Potosí.

\section{AGRADECIMIENTOS}

A los Fondos Mixtos Gobierno del Estado de San Luis Potosí-CONACYT por el apoyo financiero al proyecto $S$. L. P-2003-C02-11206, y a la Fundación Produce San Luis Potosí, A. C. por el financiamiento a los proyectos 4095703A y $2096298^{a}$.

\section{BIBLIOGRAFÍA}

Carballo C A, F Márquez S (1970) Comparación de variedades de maíz de El Bajío y la Mesa Central por su rendimiento y estabilidad. Agrociencia 5:129-146.

De León C H, F Rincón S, M H Reyes V, D S Garduño, G Martínez Z, R Cavazos C, J D Figueroa C (2005) Potencial de rendimiento y estabilidad de combinaciones germoplásmicas formadas entre grupos de maíz. Rev. Fitotec. Mex. 28: 135-143.
Eastin J D, F Zavala G (2002) Tan plant lines and populations with enhanced stress resistance. Nebraska Grain Sorghum Board Report for July 1, 2001 to June 30, 2002. University of Nebraska-Lincoln. 19 p.

Eberhart S A, W A Russell (1966) Stability parameters for comparing varieties. Crop Sci. 6:36-40.

Fisher K S, G L Wilson (1975) Studies of grain production in Sorghum bicolor (L. Moench). III. The relative importance of assimilates supply, grain growth capacity and transport system. Austr. J. Agric. Res. 26:11-23.

Fisher K S, A F E Palmer (1980) Yield efficiency in tropical maize. In: Symposium on "Potential Productivity of Field Crops Under Different Environments" IRRI, Philippines. pp:26-40.

Hernández A J A, F Zavala G, M A Martínez G, C Jasso Ch, E J Ventura R, K Durán L (2007) Tecnología para Producir Forraje de Mijo Perla en San Luis Potosí. Folleto para Productores No. 45. SAGARPA-INIFAP, México. 23 p.

Mahalakshmi V, F R Bidinger, D S Raju (1987) Effect of timing of water deficit on pearl millet (Pennisetum americanum). Field Crops Res. 14:327-339.

Maiti R K, G Gomez S (1990) Effect of four sowing date environments on growth, development and yield potential of 15 pearl millet cultivars (Pennisetum americanum L. Leeke) during autumnwinter seasons in Marín, N. L., México. J. Exp. Bot. 41:16091618.

Maiti R, U López D (1995) El Mijo Perla. Su Adaptación y Productividad. Ed. Trillas. México, D. F. 139 p.

Maman N, S C Mason, D J Lyon, P Dhuangana (2004) Yield components of pearl millet and grain sorghum across environments in the Central Great Plains. Crop Sci. 44:21382145.

Medina G G, G Díaz P, C Loredo O, V Serrano A, M A Cano G (2005) Estadísticas climatológicas básicas del estado de San Luis Potosí (periodo 1961-2001). Campo Experimental San LuisCIRNE-INIFAP. Libro Técnico No. 2. México. $321 \mathrm{p}$.

Mejía C J A, J D Molina G (2003) Cambios de estabilidad en el rendimiento de variedades tropicales de maíz. Rev. Fitotec. Mex. 26:89-94.

Muchow R C (1989) Comparativity of maize, sorghum and pearl millet in a semi-arid tropical environment II. Effect of water deficits. Field Crops Res. 20:207-219.

Olivares S F (1994) Parámetros de estabilidad (S. A. Eberhart and W. A. Russell). Universidad Autónoma de Nuevo León. Facultad de Agronomía. Marín, N. L. México. Versión 1.0 en disco compacto.

Pale S, S C Mason, T D Galusha (2003) Planting time for early-season pearl millet and grain sorghum in Nebraska. Agron. J. 95:10471053.

Presterl T, E Weltzien (2003) Exploiting heterosis in pearl millet for population breeding in arid environments. Crop Sci. 43:767-776.

Rai K N, A Kumar, D J Andrews, A S Rao, A G B Raj, J R Witcombe (1990) Registration of 'ICTP 8203' pearl millet. Crop Sci. 30:959.

Rodríguez PJ E, J Saghún C, H E Villaseñor M, J D Molina G, A Martínez G (2002) Estabilidad de siete variedades comerciales de trigo (Triticum aestivum L.) de temporal. Rev. Fitotec. Mex. 25:143-151.

Torres H, H Williams A, A S Ortegón M. (1994) El mijo perla como cultivo de alternativa para el norte de Tamaulipas. In: Memorias de la Reunión Científica y Tecnológica del Estado de TamaulipasCampo Experimental Río Bravo. Cd. Río Bravo, Tam. SARHINIFAP. México $33 \mathrm{p}$.

Villanueva D J, C Loredo O, A Hernández R (2001) Requerimientos hídricos de especies anuales y perennes en las zonas Media y Altiplano de San Luis Potosí. Folleto Técnico No. 12. Campo Experimental Palma de la Cruz. SAGARPA-INIFAP, México. 24 p. 
Vittar K P R, K Vijayalakshmi, U M V Rao (1990) The effect of cumulative erosion and rainfall on sorghum, pearl millet and castor bean yields under dryfarming conditions in Andhra Pradesh, India. Exp. Agric. 26:426-439.
Witcombe J R, M N V R Rao, A G B Raj y C T Hash (1997) Registration of 'ICMV 88904' pearl millet. Crop Sci. 37:10221023. 